\title{
Mantenimiento del peso a corto plazo en pacientes de tratamiento integral de sobrepeso y obesidad
}

ISSN 1794-9831

E-ISSN 2322-7028

Vol. 13 No. 1

Ene - Jun 2016

Cúcuta, Colombia

Recibido:

27 de Noviembre

de 2015

Zaida Rocío Contreras-Velásquez*

Tulia Isabel Copete-Villa**

Pastor Ramírez-Leal ${ }^{* * *}$

\section{RESUMEN}

Objetivo: determinar el mantenimiento del peso e índice de masa corporal en un corto plazo en pacientes entre 18 y 81 años de edad, que asistieron a tratamiento integral para sobrepeso y obesidad en consulta médica especializada. Materiales y Métodos: el diseño del estudio es de tipo descriptivo observacional. La población corresponde a 67 pacientes que asistieron a tratamiento de sobrepeso y obesidad, de la cual se seleccionó una muestra que corresponde a 53 pacientes que asistieron a terapia durante el año 2012, asistiendo a última consulta en diciembre del año 2012 y reiniciando el tratamiento en enero del 2013, a quienes se les diligenció la historia clínica, cuyos datos fueron condensados en una base de datos especialmente diseñada para el estudio. Resultados: al comparar las variables del estudio entre diciembre 2012 y enero 2013 se encontró que la media de cambio en el peso fue de $0,075 \pm 1,57 \mathrm{DS}$ ( $\mathrm{p}=0,729$ ). El porcentaje de variación de peso obtenido fue de $0,12 \%$ con DS $\pm 2,23$ y la media de la variación del índice de masa corporal fue de 0,0245 $\pm 0,62 \mathrm{DS}$ ( $\mathrm{p}=0,776$ ). Conclusión: los pacientes logran mantener peso e índice de masa corporal alcanzado en el tratamiento y mantienen éstos valores durante cortos periodos como lo es la época de navidad y fin de año, debido a que el paciente aprende a llevar los nuevos hábitos inclusive en esta corta temporada en la que la dieta, a nivel cultural, tiende a ser de alto contenido calórico.

PALABRAS CLAVE: índice de masa corporal, obesidad, sobrepeso ${ }^{* * * *}$.
Para citar este artículo / To reference this article / Para citar este artigo

Contreras-Velásquez ZR, Copete-Villa TI, Ramírez-Leal P. Mantenimiento del peso a corto plazo en pacientes de tratamiento integral de sobrepeso y obesidad. Rev. cienc. cuidad. 2016; 13(1): 43-58.
Aceptado por

pares: 26 de

Febrero de 2016

Aprobado:

5 de Abril de 2016

*Bacterióloga.

Especialista en

Epidemiología

Clínica. Especialista

en Prácticas

Pedagógicas

Universitarias.

Docente. Universidad

Francisco de

Paula Santander.

Cúcuta, Colombia.

Correo electrónico:

zaidarociocv@ufps.

edu.co

** Médico.

Especialista en

Medicina Estética.

Coordinador médico

y científico del

Instituto Medico

Científico de Estética

Avanzada IMCEA.

Cúcuta, Colombia.

Correo electrónico:

tuliacopete@hotmail.

com

*** Licenciado

en Matemáticas

y Computación.

Especialista

en Estadística

Aplicada. Magister

en Educación

Matemática. Docente.

Universidad

Francisco de

Paula Santander.

Cúcuta, Colombia.

Correo electrónico:

pastorramirez@ufps.

edu.co

****Descriptores en

Ciencias de la Salud

(DeCS), en la página

http://decs.bvs.br/E/

DeCS2015_Alfab-S.

$\mathrm{htm}$ de la Biblioteca

virtual en salud del proyecto BIREME,

de la Organización

Mundial de la Salud

y de la Organización

Panamericana de la

Salud. 
ISSN 1794-9831

E-ISSN 2322-7028

Vol. 13 No. 1

Ene - Jun 2016

Cúcuta, Colombia

\section{Short-term weight management in the comprehensive treatment of excess weight and obesity in patients}

\section{ABSTRACT}

Objective: to determine the short-term weight management and body mass index in patients between 18 and 81 years old, who attended specialized medical consultation for excess weight and obesity comprehensive treatment Methods: the study design is of a observational descriptive type. The population involves 67 patients who attended overweight and obesity treatment, of which a sample of 53 patients attended therapy during 2012 taking part in the last consultation in December 2012 and restarting the treatment in January 2013, whose medical history was filled out and whose data were also condensed into a database specifically designed for the study. Results: when comparing the study variables between December 2012 and January 2013, it was found that the average weight change was 0.075 $\pm 1,57 \mathrm{DS}(\mathrm{p}=0.729)$. The $\%$ weight change was $0.12 \%$ obtained with $\mathrm{DS} \pm 2.23$ and the mean change of body mass index of $0.0245 \pm$ was $0,62 \mathrm{DS}(\mathrm{p}=0.776)$. Conclusion: patients are able to maintain weight and body mass index achieved in treatment, and maintain these values for short periods such as the Christmas season and New Year, this is so because the patient learns to adopt new habits even in this short season when one's diet, culturally, tends to be high in calorie.

KEYWORDS: body mass index, obesity, overweight. 


\section{Manutenção de peso a curto prazo em pacientes de tratamento abrangente de sobrepeso e obesidade}

\section{RESUMO}

Objetivo: determinar a manutenção do peso e índice de massa corporal, a curto prazo em pacientes entre 18 e 81 anos de idade, que freqüentavam o tratamento abrangente para sobrepeso e obesidade em materiais especializados consulta médica. Materiais e Métodos: o desenho do estudo é do tipo observacional descritivo. A população corresponde a 67 pacientes que frequentam o tratamento do sobrepeso e da obesidade, que uma amostra correspondente a 53 pacientes submetidas à terapia em 2012 participando última visita em dezembro de 2012 e reiniciar o tratamento em janeiro foi selecionado 2013, que eram eu enchi a história médica, cujos dados foram condensados em um banco de dados projetado especificamente para o estudo. Resultados: ao comparar as variáveis de estudo entre Dezembro de 2012 e Janeiro de 2013, verificou-se que a mudança de peso foi de $0.075 \pm$ $1,57 \mathrm{DS}(\mathrm{p}=0,729)$. A mudança de peso $\%$ foi de $0,12 \%$ obtida com $\mathrm{DS} \pm 2,23$ e a variação média do índice de massa corporal de $0,0245 \pm$ era $0,62 \mathrm{DS}(\mathrm{p}=0,776)$. Conclusão: os pacientes são capazes de manter o peso e índice de massa corporal alcançado em tratamento, e manter esses valores para períodos curtos, como a época do Natal e Ano Novo, isto porque o paciente aprende a assumir novos hábitos, mesmo em esta curta temporada que a dieta, culturalmente, tende a ser alto teor calórico.

PALAVRAS-CHAVE: índice de massa corporal, obesidade, sobrepeso. 
ISSN 1794-9831

E-ISSN 2322-7028

Vol. 13 No. 1

Ene - Jun 2016

Cúcuta, Colombia

\section{INTRODUCCIÓN}

egún la Organización Mundial de la Salud OMS (1), el sobrepeso y la obesidad se definen como una acumulación anormal o excesiva de grasa que puede ser perjudicial para la salud. El índice de masa corporal (IMC) es un indicador simple de la relación entre el peso y la talla que se utiliza frecuentemente para identificar el sobrepeso y la obesidad en los adultos y se calcula dividiendo el peso de una persona en kilos por el cuadrado de su talla en metros $\left(\mathrm{Kg} / \mathrm{m}^{2}\right)$.

La definición es la siguiente:

- Un IMC igual o superior a 25 es indicativo en sobrepeso.

- Un IMC igual o superior a 30 es indicativo a obesidad.

Según Lastra y Lastra (2), la obesidad es una de las enfermedades metabólicas más antiguas de la humanidad; sin embargo, hasta hace relativamente poco se ha reconocido como tal. La Organización Panamericana de la Salud (OPS), en 2008, ya contaba con 1500 millones de adultos (de 20 años o más) que tenían sobrepeso. Dentro de este grupo, más de 200 millones de hombres y cerca de 300 millones de mujeres eran obesos.

Algunas estimaciones mundiales recientes de la OMS (1) son las siguientes:

- En 2014, más de 1.900 millones de adultos de 18 o más años tenían sobrepeso, de los cuales, más de 600 millones eran obesos.

- En general, en 2014 alrededor del $13 \%$ de la población adulta mundial, un $11 \%$ de los hombres y un $15 \%$ de las mujeres, eran obesos.

- En 2014, el $39 \%$ de los adultos de 18 o más años $(38 \%$ de los hombres y un $40 \%$ de las mujeres) tenían sobrepeso.

- La prevalencia mundial de la obesidad se ha multiplicado por más de dos entre 1980 y 2014.

La causa fundamental del sobrepeso y la obesidad es un desequilibrio energético entre calorías consumidas y gastadas, que en la mayoría de los países de América
Latina y el mundo se ha generado por un cambio en los hábitos alimentarios, con un vertiginoso aumento del consumo de alimentos con un denso contenido energético, ricos en grasas saturadas, azúcares y sal.

Según Alberto Barceló, asesor regional de enfermedades no transmisibles de la Organización Panamericana de la Salud (3), los malos hábitos alimenticios, unidos a una creciente cultura de sedentarismo y falta de actividad física, están contribuyendo gravemente al incremento en las tasas de sobrepeso y obesidad en la región. La prevalencia de la obesidad está aumentando en todos los grupos de edad en América, así como en otras regiones del mundo.

Gómez (4), al comentar el libro Sobrepeso y Obesidad: epidemiología, evaluación y tratamiento, opina, entre otras cosas, que el individuo obeso crece en condiciones que no le dejan mucha opción. La comida que se le ofrece desde pequeño le hace subir de peso, anexo a las opciones que hoy en día se tienen, que son de muy mala calidad alimenticia. En este aspecto, existe un fenómeno social que incrementa la obesidad al mismo tiempo que identifica la comida industrializada como un bien deseable para todo evento y circunstancia.

De acuerdo a Pereira y Palay (5), la obesidad es un trastorno metabólico y nutricional de serias consecuencias para la salud. Aunque existe un mejor conocimiento clínico y epidemiológico del problema, la prevalencia de la obesidad ha aumentado significativamente debido, en parte, a que la ingesta calórica es superior al gasto energético, lo cual finalmente lleva a un aumento de la grasa corporal.

Así mismo, la OMS (1) establece que a nivel mundial, en las últimas décadas, se ha presentado un notable incremento de las enfermedades crónicas no transmisibles (ECNT) asociadas a estilos de vida no saludables. Estas enfermedades y trastornos en conjunto, entre las que se encuentran las cardiovasculares, como lo plantea De Marco et al. (6) -principalmente cardiopatía y accidente cerebrovascular-, la diabetes, los trastornos del aparato locomotor (en especial osteoartritis) y algunos cánceres (endometrio, mama y colon), son las principales causas de muerte, discapacidad y deterioro de la calidad de vida. 
Herrera et al. (7), afirman que recientemente se ha comenzado a considerar a la obesidad como un factor de riesgo más en el desarrollo del cáncer, pues se correlaciona con una probabilidad de aparición de esta enfermedad. Aunque las causas exactas que vinculan estos dos padecimientos de salud pública no están del todo esclarecidas, algunos investigadores sugieren que entre el $35 \%$ y el $70 \%$ de los cánceres están vinculados directamente con la alimentación y con un IMC elevado que refleja obesidad. La OMS (1) sugiere que el riesgo de contraer estas enfermedades no transmisiblescrece con el aumento del IMC.

La Secretaría de Salud de Santander (8), asevera que en Colombia y países de ingresos bajos y medianos las enfermedades no transmisibles presentan una elevada carga de morbi-mortalidad, junto con las enfermedades infecciosas y la desnutrición; inclusive, no es raro encontrar coexistiendo desnutrición y obesidad en un mismo país, comunidad o hasta en un mismo hogar.

Así mismo, Lastra y Lastra (2) explican que debido a su alta prevalencia, impulsada por un estilo de vida basado en la sobrealimentación y en el sedentarismo, se ha convertido en la epidemia de este milenio, un problema de salud pública que en algunos países representa un alto porcentaje del gasto social.

La OMS (1) también menciona que la responsabilidad en la prevención del sobrepeso y la obesidad van desde el plano individual, limitando la ingesta de grasa total y azúcares, aumentando el consumo de frutas, verduras, legumbres, cereales integrales y frutos secos, además de la realización de actividad física periódica; hasta el plano social, en el cual es importante el apoyo a las personas para el cumplimiento de las recomendaciones antes mencionadas, mediante un compromiso político sostenido y la colaboración de las múltiples partes interesadas, públicas y privadas, así como lograr que la actividad física periódica y los hábitos alimenticios sean más saludables y económicamente accesibles para todos, en particular para las personas más pobres.

Para Amzallag (9), mientras la mayoría de los candidatos quieren adelgazar con fines estéticos, los especialistas subrayan como más importante la mejoría de las complicaciones relacionadas con la obesidad. De un modo más específico, se puede decir que los objetivos del tratamiento de la obesidad se han trasladado de la sencilla pérdida de peso aislada como meta estética, hacia una gestión global de una mejor u optima salud.

Este nuevo concepto de Amzallag (9) incluye la pérdida de peso pero no se limita a esto. En consecuencia, un programa de gestión para la pérdida de peso puede ser considerado como eficaz si el paciente responde favorablemente a una o varias de las siguientes estrategias:

- La prevención de la gordura.

- La mejoría de las complicaciones asociadas.

- La mejoría de las reglas generales de salud e higiene de la vida.

- El adelgazamiento estable a largo plazo.

Alcanzar sus objetivos es responsabilidad conjunta del paciente y del profesional de la salud al saber que, cualquiera sea el valor del programa, sólo el paciente puede ponerlo en práctica.

Según Warburton et al. (10), existe una amplia y contundente evidencia científica que demuestra que las personas físicamente activas, en comparación con las que no lo son, poseen menores tasas de mortalidad, enfermedad coronaria, hipertensión arterial, accidente cerebrovascular, diabetes tipo 2 , síndrome metabólico, cáncer de colon, cáncer de mama y depresión, además de presentar una composición corporal más saludable y un perfil de biomarcadores más favorable para la prevención de enfermedad cardiovascular.

Según Malik etal. (11), debido a la amplia complejidad de la epidemia de la obesidad se necesitan estrategias políticas con múltiples niveles de prevención que, a la final, permitan tener un efecto medible en la sociedad. Los cambios deben incluir políticas globales de alto nivel en la comunidad internacional y los esfuerzos coordinados de gobiernos, organizaciones, comunidades e individuos para influir positivamente en el cambio de comportamiento.

En Colombia, la situación no es mejor. Según la última Encuesta Nacional de la Situación Nutricional (ENSIN) (12), llevada a cabo en 2010, se evidencia el incremento en los indicadores de obesidad: subió del $48 \%$ al $52 \%$ con un índice más alto para las mujeres. 
El indicador es de $45 \%$ para los hombres y el $55 \%$ para las mujeres.

Esta misma encuesta arrojó que el exceso de peso en la población adolescente, adulta y gestante muestra una tendencia al incremento y afecta a las dos terceras partes de los grupos de mayor edad, a pesar de los esfuerzos realizados para su prevención (12). También determinó que el 46,5\% de la población de adultos entre 18 y 64 años es físicamente inactivo.

En materia de legislación existe la Ley 1355 de 2009 (13), la cual define la obesidad y las enfermedades crónicas no transmisibles asociadas como una prioridad de salud pública. Con esta ley se busca promover ambientes sanos, actividad física, educación, producción y distribución de alimentos con previa regulación de grasas trans y grasas saturadas.

Aun así, las políticas de salud en materia de control y mantenimiento del peso no son establecidas por las empresas que prestan un servicio de salud a la población; no existe una intervención significativa en los hábitos de las personas que empiezan a presentar síntomas de la enfermedad y, por lo tanto, la mayoría de las veces las consecuencias de la enfermedad no se previenen a tiempo.

Desde el punto de vista clínico se han propuesto diferentes tipos de métodos terapéuticos con el fin de lograr la reducción de peso. En el estudio de Martínez et al. (14) se evalúa la eficacia en la reducción de peso con la utilización de dieta de muy bajo aporte calórico cetogénica y una posterior reeducación alimentaria según el método PronoKal ${ }^{\circledR}$ en pacientes con sobrepeso y obesidad y la seguridad durante su aplicación.

\section{MATERIALES Y MÉTODOS}

La investigación se originó por una necesidad sentida del Coordinador Médico Científico de la institución $\mathrm{X}$ objeto de estudio, quien, una vez planteó su inquietud, permitió de manera voluntaria el acceso a la base de datos de dicha institución, dando así inicio a la investigación.

El diseño del estudio fue de tipo descriptivo observacional, de corte retrospectivo. Para ello se seleccionó una población, conformada por 67 pacientes, entre hombres y mujeres, quienes recibieron tratamiento integral de sobrepeso y obesidad durante el año 2012. De esta población se seleccionó una muestra a conveniencia del investigador, que correspondió a 53 pacientes que durante la terapia, en el año 2012, asistieron a una última consulta en diciembre de 2012 y reiniciaron el tratamiento en enero de 2013 en la institución X. Según los criterios del estudio, los pacientes que harían parte de la muestra debían ser mayores de 18 años, con registros de historia clínica completa.

Los pacientes que asistieron a consulta tenían cita previa programada y fue a quienes se les diligenció la historia clínica en la cual se consignaron datos demográficos (género, actividad económica, edad, etc.) y clínicos (peso, índice de masa corporal y perímetro abdominal) de cada uno, según lo establecido en diversos estudios referenciados por Amzallag (9), Xiao et al. (15), Yank et al. (16), Martínez (17) y Moreno (18).

Con el fin de mantener los principios bioéticos en la investigación, a todos los pacientes, previo a la solicitud del consentimiento informado, se les proporcionó una explicación de la condición del tratamiento, objetivos, posibles beneficios, riesgos, incomodidades derechos y responsabilidades, de acuerdo con lo establecido en la actual Declaración de Helsinki (19).

Durante el tratamiento se practicó una exploración a los pacientes en busca de enfermedades endocrinas relacionadas con el exceso de peso. El paciente debió consignar su estilo de alimentación y ejercicios habituales. Con la información condensada se inició un manejo integral individual con terapia médica nutricional y diversos procedimientos médicos estéticos, de acuerdo con los antecedentes patológicos del paciente y control semanal.

De esta manera se realizó un control progresivo, continuo y constante del paciente que permitió detectar posibles errores en el cumplimiento de su dieta, así como una mejor adaptación a los diferentes procedimientos.

Con el fin de realizar una selección adecuada de la muestra, los datos obtenidos de las historias clínicas fueron consignados, en su totalidad, en un una base de datos diseñada en Excel, específicamente para este 
estudio. En dicha base de datos se tuvieron en cuenta, de manera principal, las medidas antropométricas -peso, índice de masa corporal y perímetro abdominal-.

Las variables directamente relacionadas con el estudio fueron: el peso $(\mathrm{kg})$ al iniciar el tratamiento en el 2012, peso en el último control de 2012 y peso en el primer control de 2013, variación de peso $(\mathrm{kg})$, porcentaje de variación de peso, índice de masa corporal (IMC), variación del índice de masa corporal y perímetro abdominal. Otras variables analizadas fueron edad, género y ocupación, entre otras. Posterior a la recolección de la información, se realizó el análisis en el programa SPSS, versión 21.

Además, serealizó control de sesgos a las variables peso e IMC por medio de pruebas paramétricas (Shapiro Wilk), con valor $p>0,05$, por lo que se estableció que las variables presentan el comportamiento de una curva de distribución normal.

Se determinó, por prueba de hipótesis, la comparación de medias para variables continuas por medio de la $t$ de student y prueba de Wilcoxon para muestras pareadas, esto con el fin de determinar si se observaba diferencia de medias entre la primera consulta de 2012 vs consulta de diciembre de 2012 y última consulta de 2012 vs primera consulta de 2013 . Se tuvo en cuenta un intervalo de confianza del $95 \%$ en cada uno de los análisis. Igualmente, se hizo la correlación con el valor de significancia (valor $\mathrm{p}$ ) considerando que las diferencias eran estadísticamente significativas para una $\mathrm{p}<0,05$.

Igualmente, se comparó la media obtenida en la variación del peso, variación del índice de masa corporal y el porcentaje de variación de peso entre la primera consulta de 2012 y la consulta obtenida en diciembre de 2012 con los resultados del estudio de Xiao et al. (15), teniendo en cuenta que la muestra utilizada por éste estuvo monitoreada por entrenadores especializados (coach), tratamiento terapéutico similar al realizado en la institución X donde se llevó a cabo el estudio mediante un control periódico de medidas a los pacientes durante todo el tratamiento.

\section{OBJETIVOS}

\section{Objetivo general}

Determinar el mantenimiento del peso e índice de masa corporal (IMC) en corto plazo, en pacientes de tratamiento integral para sobrepeso y obesidad.

\section{Objetivos específicos}

- Determinar las características demográficas y epidemiológicas de la población que asiste al programa de manejo integral de sobrepeso y obesidad.

- Identificar los antecedentes clínicos de importancia al iniciar el programa de manejo integral de sobrepeso y obesidad.

- Determinar el mantenimiento de las variables peso, índice de masa corporal (IMC) y perímetro abdominal en los tres momentos del estudio.

\section{RESULTADOS}

\section{Características sociodemográficas}

Las características sociodemográficas de la muestra estudiada, correspondientes a la primera consulta del año 2012, son las siguientes:

Del total de la muestra, el paciente de menor edad tenía 18 años y el de mayor edad tenía 83 años; así mismo, la media se encontraba en 43,45 años con un $\mathrm{DS} \pm 12,35$ y un IC $95 \%(40,12-46,77)$. De manera significativa, se evidenció mayor asistencia del género femenino a la consulta del especialista en medicina estética $(90,6 \%)$, entre las cuales predominó el estado civil casado en un $52,8 \%$.

El estado civil en el cual se encuentra el 52,8 \% de los pacientes es casado, seguido del estado civil soltero con un 26,4 \%. Le siguen unión libre con un 9,4 \% y separado y viudo, cada uno con $5,7 \%$, como se puede observar en la figura 1. 
ISSN 1794-9831

E-ISSN 2322-7028

Vol. 13 No. 1

Ene - Jun 2016

Cúcuta, Colombia
Figura 1.Distribución de acuerdo al estado civil.

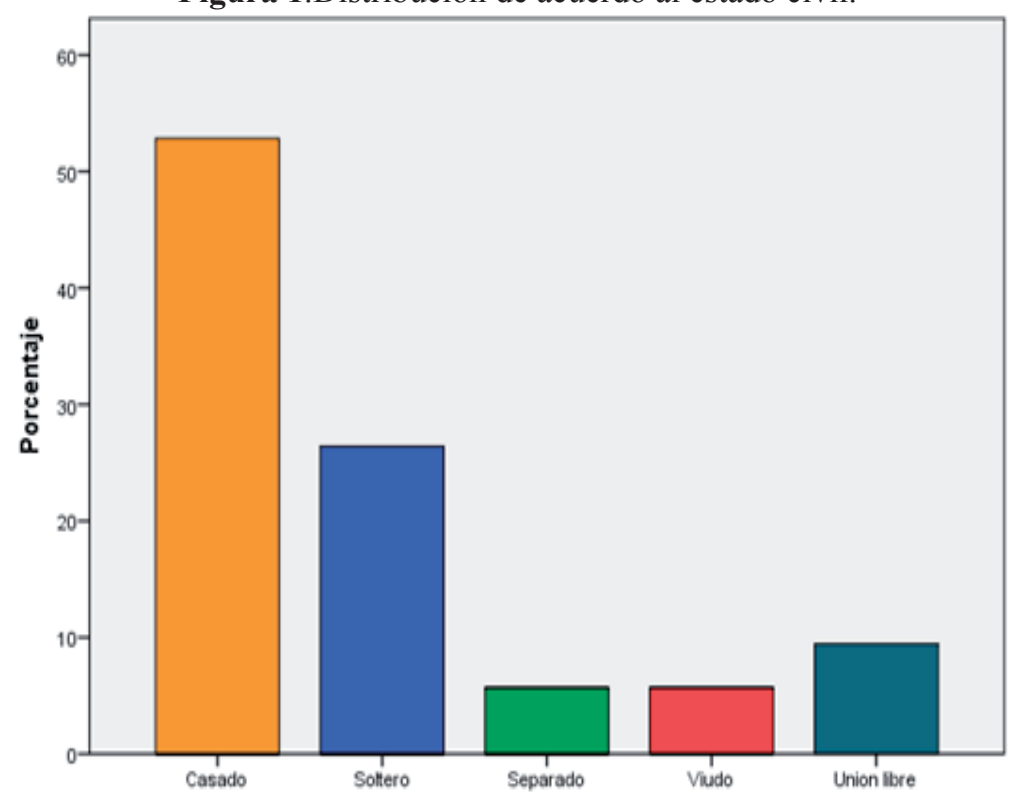

Fuente: Base de Datos de Pacientes con manejo integral de sobrepeso y obesidad, 2013.

En la figura 2 se muestra la distribución de la ocupación y la que más relevancia tuvo en la muestra analizada fue finanzas y administración con un 26,4

$\%$, seguida de hogar con un $13,2 \%$ en ventas y servicios, mientras que la ocupación relacionada con la salud presentó un $9,4 \%$.

Figura 2: Distribución de acuerdo a la ocupación.

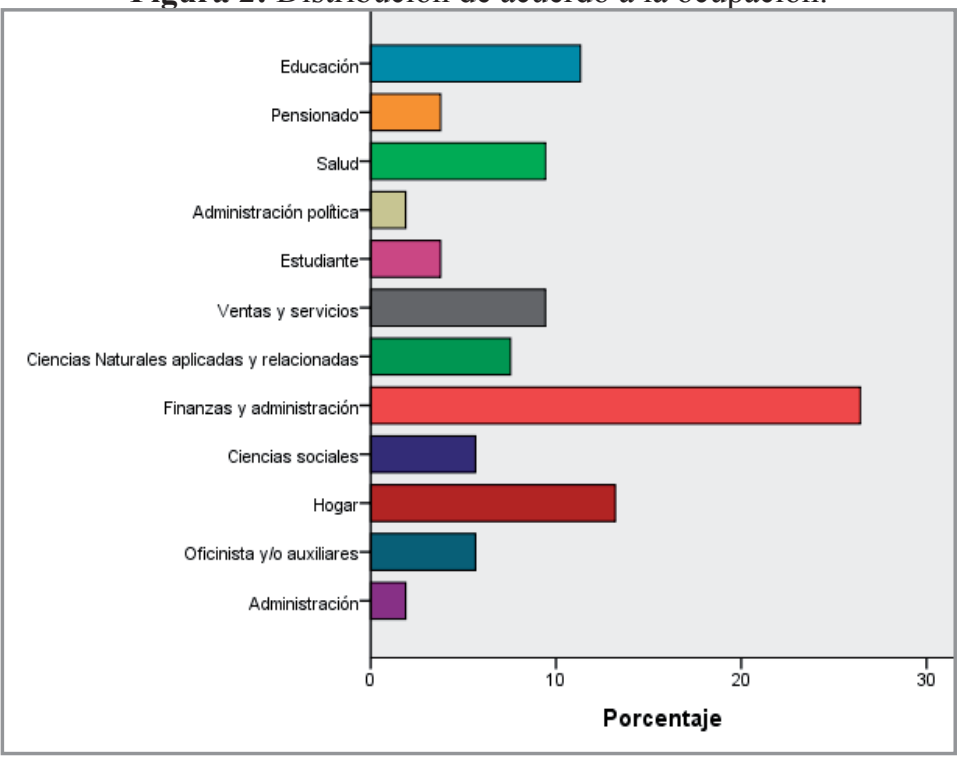

Fuente: Base de Datos de Pacientes con manejo integral de sobrepeso y obesidad, 2013. 


\section{Antecedentes clínicos}

En la primera consulta se realiza una valoración médica, la cual incluye los antecedentes clínicos del paciente, con el fin de determinar el tratamiento más pertinente. Entre las variables de importancia se encuentra enfermedades de base, antecedentes clínicos de cirugías relacionadas con moldeamiento (liposucción, dermolipectomía), antecedentes de cirugías ortopédicas y hábito de ejercicio.

Según la figura 3 , un $26,4 \%$ de las pacientes del estudio manifestó haberse realizado algún tipo de cirugía relacionada con moldeamiento.

Figura 3. Antecedentes clínicos de cirugías (\%).

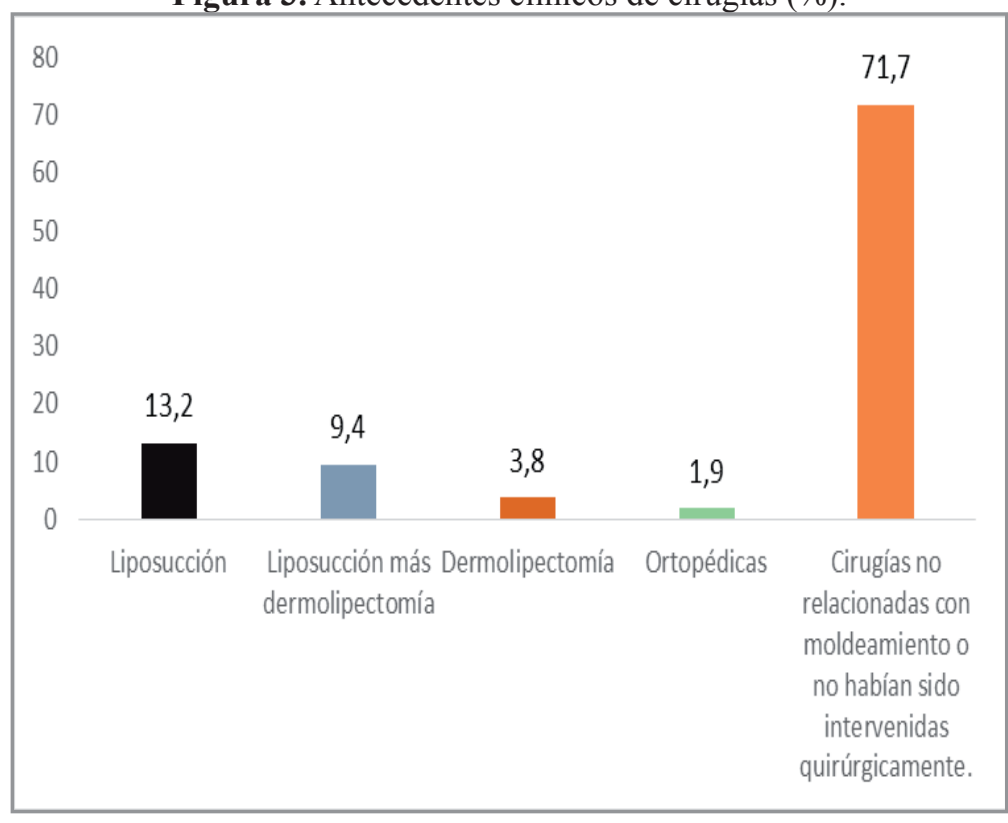

Fuente: Base de Datos de Pacientes con manejo integral de sobrepeso y obesidad, 2013.

El no tener hábito de ejercicio, en la primera consulta, predominaba en un 58,5\%. Así mismo, entre los antecedentes de importancia clínica predominantes se encontraba la dislipidemia en un $16,7 \%$ y la hipertensión arterial en el 13,6\%.

\section{Características de las variables en los tres momentos del estudio}

Los valores obtenidos de peso, índice de masa corporal y perímetro abdominal, durante los tres momentos del estudio, incluyeron los resultados obtenidos al inicio del tratamiento para sobrepeso y obesidad, diciembre del 2012 y primera consulta del 2013, así:

El peso, al inicio del tratamiento, obtuvo una media de $76,35 \mathrm{~kg}$ con $\pm 14,35$ DS IC $95 \%(72,48-80,20)$; en el último control del mes de diciembre de 2012
ISSN 1794-9831

E-ISSN 2322-7028

Vol. 13 No. 1

Ene - Jun 2016

Cúcuta, Colombia tuvo una media de $70,50 \mathrm{~kg}$ con $\pm 11,1$ DS IC $95 \%$ $(67,51-73,48)$ y en el primer control del año 2013 la media del peso en $\mathrm{kg}$ se encontró en $70,57 \mathrm{~kg}$ con una DS $\pm 11,205$ IC $95 \%(67,55$ - 73,58).

Los datos correspondientes al IMC no presentaron una curva de distribución normal, corroborado por la prueba de Shapiro Wilks. La mediana, en la primera consulta de 2012 , se encontraba en $28,5 \mathrm{~kg} / \mathrm{m} 2$; en la consulta de diciembre de 2012 ésta era de 27,00 $\mathrm{kg} / \mathrm{m} 2$ y en la primera consulta del año 2013 la mediana se encontraba en $26,7 \mathrm{~kg} / \mathrm{m} 2$. Tan sólo, en diciembre del 2012, el P25 del IMC se encontraba en $23,9 \mathrm{Kg} / \mathrm{m} 2$, lo cual fue indicativo de un leve cambio de sobrepeso a peso normal en el tratamiento correspondiente a la primera consulta y la consulta en diciembre, en un pequeño número de pacientes que asistieron a consulta con el especialista tratante. 
ISSN 1794-9831

E-ISSN 2322-7028

Vol. 13 No. 1

Ene - Jun 2016

Cúcuta, Colombia
El perímetro abdominal o circunferencia abdominal presentó, en la primera consulta de 2012, una media (X) de $95,15 \mathrm{~cm} \pm 14,5$ DS IC $95 \%(91,42-99,28)$; en la consulta del mes de diciembre de 2012 la media (X்) del perímetro abdominal correspondió a 89,81 $\mathrm{cm} \pm 12,17$ DS IC $95 \%(86,61-93,19)$ y en la consulta del mes de enero de 2013 la media $(\dot{X})$ del perímetro abdominal se encontró en $90,27 \mathrm{~cm} \pm 12,05$ DS IC $95 \%(87,18-93,53)$.

En la tabla 1 se presentan los valores obtenidos de la variación de peso, porcentaje de variación de peso, variación del índice de masa corporal y variación del perímetro abdominal entre la primera consulta de 2012 y la consulta de diciembre de 2012, así como la última consulta de 2012 y la primera consulta de 2013.

Las fórmulas utilizadas para obtener los datos nombrados anteriormente fueron las siguientes:

- Variación de peso $=$ Peso final - peso inicial

- $\%$ de Variación de peso $=$ (Variación de peso/peso inicial) * 100

- $\quad$ Índice de masa corporal $($ IMC) $=$ Peso $(\mathrm{Kg}) /$ Talla $(\mathrm{cm})^{2}$

- Variación del IMC $=$ IMC final - IMC inicial

Tabla 1. Valores obtenidos de la variación del peso, índice de masa corporal, perímetro abdominal y \% de variación de peso entre la primera consulta de 2012 a la última consulta de diciembre de 2012 comparado con la última consulta de diciembre de 2012 a la primera de 2013.

\begin{tabular}{|l|c|l|c|c|}
\hline \multicolumn{1}{|c|}{ Variables } & $\begin{array}{c}\text { Primera consulta 2012 } \\
\text { a última consulta en } \\
\text { diciembre 2012 }\end{array}$ & $\begin{array}{l}\text { Valor } \\
\text { de p }\end{array}$ & $\begin{array}{c}\text { Última consulta en } \\
\text { diciembre 2012 a } \\
\text { primera consulta 2013 }\end{array}$ & Valor de p \\
\hline Variación del peso (kg) & $-5,8 \pm 5,0$ & 0,000 & $-0,075 \pm 1,58$ & 0,729 \\
\hline $\begin{array}{l}\text { Porcentaje de } \\
\text { variación del peso }\end{array}$ & $-7,05 \pm 5,6$ & $\begin{array}{c}\text { No se } \\
\text { obtiene* }\end{array}$ & $0,12 \pm 2,23$ & $\begin{array}{c}\text { No se } \\
\text { obtiene* }\end{array}$ \\
\hline $\begin{array}{l}\text { Variación del índice de } \\
\text { masa corporal (IMC) } \\
\left(\mathrm{Kg} / \mathrm{cm}^{2}\right)\end{array}$ & $-1,7$ en P25-50 & $0,000^{* *}$ & $0,024 \pm 0,62$ & 0,774 \\
\hline $\begin{array}{l}\text { Variación del } \\
\text { perímetro abdominal } \\
(\mathbf{c m})\end{array}$ & $-4,5$ en P25-50 & $0,000^{* *}$ & $0,46 \pm 0,17$ & 0,010 \\
\hline
\end{tabular}

Fuente: Base de Datos de Pacientes con manejo integral de sobrepeso y obesidad, 2013.

* No es posible obtener el valor p del porcentaje de variación del peso por no tener una muestra para correlacionar al ser obtenido este valor un cambio porcentual, el cual corresponde a un cociente cuyo numerador se constituye con el cambio entre dos momentos y el denominador es un cambio de base.

**Prueba de Wilcoxon de los rangos con signos de muestras relacionadas. Se muestran significancias asintóticas. El nivel de significancia es 0,05 .

Cabe recalcar que se presenta variación del peso, IMC y perímetro abdominal entre la primera consulta de 2012 y la consulta de diciembre del $2012(\mathrm{p}=0,000)$; a diferencia de la variación del peso $(\mathrm{p}=0,729)$ e IMC $(p=0,774)$ entre la consulta de diciembre de 2012 y la primera consulta del 2013.

La media de variación del peso obtenido entre la primera consulta de 2012 y la consulta de diciembre fue de $-5,85 \pm 5,0 \mathrm{~kg}$ IC $95 \% 4,47-7,23$. El porcentaje de variación de peso obtenido entre la primera consulta de 2012 y la última consulta de diciembre de 2012 obtenido fue de $-7,5 \pm 5,5 \% \mathrm{y}$, finalmente, la variación del IMC entre la primera consulta de 2012 y la consulta de diciembre de 2012 estaba en $-1,7 \mathrm{~kg} / \mathrm{m} .2$.

Una breve comparación de la variación del peso obtenido en los tres momentos del estudio se puede evidenciar en la Figura 4. 
Figura 4. Comparación y nivel de significancia entre la media del peso en los tres momentos del estudio y la media de variación del peso.

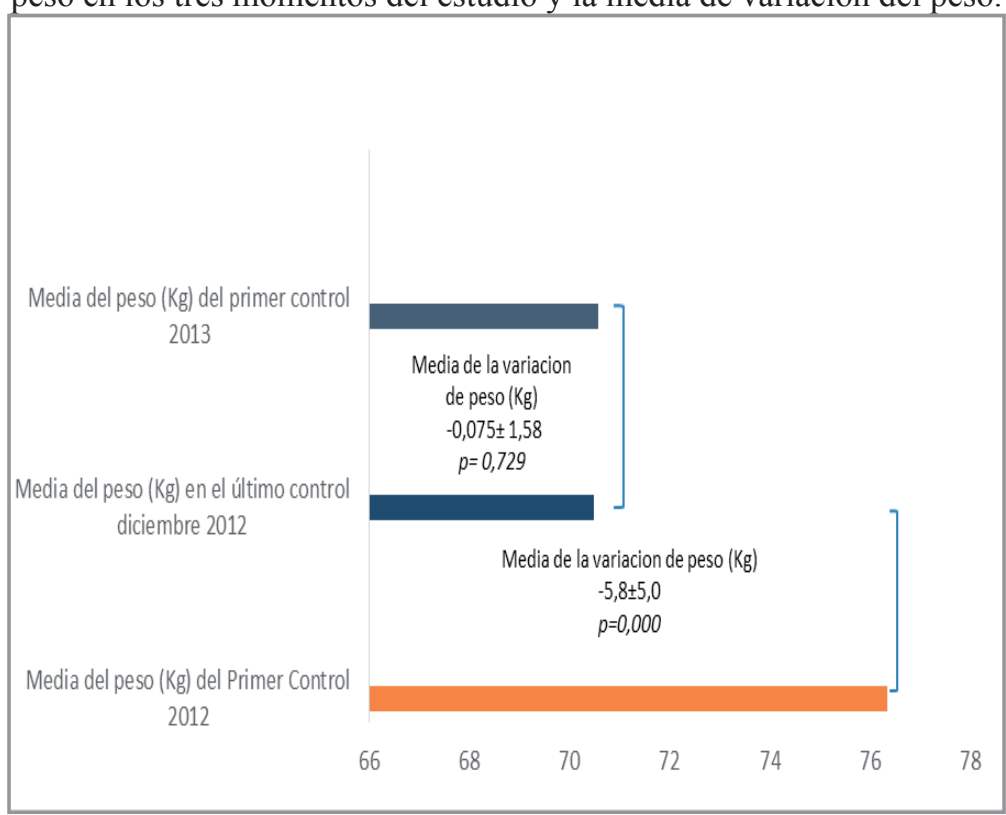

Fuente: Base de Datos de Pacientes con manejo integral de sobrepeso y obesidad, 2013.
ISSN 1794-9831

E-ISSN 2322-7028

Vol. 13 No. 1

Ene - Jun 2016

Cúcuta, Colombia

\section{DISCUSIÓN}

Para lograr una disminución significativa del peso, índice de masa corporal y riesgo cardiovascular (dependiente del perímetro abdominal) es indispensable la intervención por parte de un profesional en hábitos alimenticios y actividad física, con el fin de alcanzar el peso e índice de masa corporal ideal, así como lograr el mantenimiento de medidas a largo plazo. De igual forma, el paciente debe adquirir el compromiso de asumir la responsabilidad del tratamiento en cortos periodos de tiempo, en especial cuando, de manera cultural, se encuentra expuesto al consumo de alimentos con alto contenido calórico y, de manera simultánea, no cuenta con el acompañamiento del profesional.

El estudio también permite evidenciar que los pacientes logran disminuir significativamente el peso, IMC y perímetro abdominal durante un periodo largo si se encuentran sometidos a un entrenamiento que, a su vez, esté supervisado de manera permanente y constante por una persona especializada en manejo de hábitos (también llamado coach).

Para los investigadores del estudio fue de suma importancia determinar si los pacientes, en periodos cortos de tiempo, lograban mantener el peso que habían perdido durante el tratamiento. Con el presente estudio se demostró que los pacientes en este periodo corto de tiempo (entre la última consulta de diciembre de 2012 y la primera consulta del año 2013) lograron un mantenimiento significativo del peso $(p=0,729)$ e IMC ( $\mathrm{p}=0,774)$, lo cual demuestra que los pacientes, de manera adecuada, llevaron a cabo la dieta y la actividad física que previamente había sido recetada por el profesional. Lo anterior permite establecer que es posible el mantenimiento del peso e IMC durante cortos periodos de tiempo, inclusive si éstos son considerados por los pacientes como difíciles, dada la alta frecuencia en el consumo de alimentos ricos en calorías que ocurre en las épocas de navidad y fin de año.

Se han realizado estudios similares como el de Yank et al. (16), quienes observaron una muestra de 72 pacientes que fueron intervenidos en el estilo de vida, y donde se analizaron los patrones de pérdida de peso después de un corto periodo de tiempo (12 semanas); a su vez se evaluaron las diferencias en el mantenimiento de la pérdida de peso a los 15 meses de tratamiento. Se encontraron tres patrones de pérdida de peso, una modesta ( $21 \%)$, moderada y estable (60 $\%$ ) y sustanciales y temprana (19\%). 
ISSN 1794-9831

E-ISSN 2322-7028 Vol. 13 No. 1

Ene - Jun 2016 Cúcuta, Colombia
Yank et al. (16), encontraron que el cambio más significativo de porcentaje de pérdida de peso se evidenció en aquellos que perdieron más peso durante el tiempo estipulado; así mismo, este porcentaje de pérdida de peso aumentó significativamente, inclusive hasta el final del tratamiento, a diferencia de los pacientes cuyo porcentaje de disminución de peso no fue significativo.

De otro lado, de acuerdo con los datos del estudio, aunque la media de disminución del peso a corto plazo se encontraba en $-0,075 \pm 1,58$, es necesario especificar que algunos pacientes aumentaron de peso, otros lo mantuvieron y algunos lograron disminuirlo en este periodo de tiempo, lo cual indica que es importante la asistencia inmediata al especialista, con el fin de no ver alterado el resultado obtenido en el tratamiento.

Al respecto, Amzallag (9) menciona la importancia de que el paciente se mantenga en un programa que incluya un régimen dietético hipocalórico de manera continua, evitar la variación cíclica de peso (dietas tipo yo-yo), una restricción suficiente para producir un déficit de energía -lo cual debe incluir el ejercicio-, un equilibrio de nutrientes que mantenga unos requerimientos proteicos mínimos, un nivel de carbohidratos entre 30 a 50 gramos diarios y un programa de control del peso; todo lo anterior con el fin de alcanzar una buena mejoría de la salud representada en la reducción del colesterol sanguíneo, mejoría en la presión sanguínea, aumento de la diuresis (al inicio de la dieta) y mejoramiento de la diabetes tipo 2 .

En los pacientes de la presente investigación se evidenció una pérdida de medidas en el perímetro abdominal, como se muestra en la Tabla 4, tema que ha sido motivo de estudio de Martínez (17) y Moreno (18). Moreno (18) sugiere incluir trabajos locales que permitan establecer puntos de corte para cada región con el fin de determinar, con mejor precisión, los valores de riesgo y presenta un estudio de la Encuesta de Caracterización Socioeconómica Nacional de Chile, en el cual se demuestra una relación entre la prevalencia de hipertensión arterial con cifras de perímetro abdominal más altos y sugiere incluir el hábito de medición del perímetro abdominal como herramienta de estudio del riesgo cardiometabólico.

El estudio de meta -análisis de Johansson et al. (20), observó mejoría en el mantenimiento de la pérdida de peso después de una combinación de dieta muy baja en calorías, acompañada de medicamentos contra la obesidad y sustitutos de las comidas, dieta alta en proteínas como aporte a posibles tratamientos para la obesidad; aun así, sugiere más trabajos que permitan aclarar el efecto de la combinación de varias estrategias de mantenimiento del peso que permita mejorar los programas de tratamiento de la obesidad.

En el presente estudio, al inicio del tratamiento, se preguntó por el hábito de realizar ejercicios y más del $50 \%$ de los pacientes manifestó tener este hábito. La deficiencia de este hábito, sumado a otros hábitos como mirar televisión por largo tiempo, mantenerse sentado en el trabajo y el uso del automóvil como medio de transporte, aumenta la probabilidad de padecer enfermedades como son la diabetes tipo 2, según afirma la Revista Panamericana de la Salud (21); es por ello que resulta necesario incluir un programa de ejercicios o aumento de actividad física al inicio del tratamiento, que se adapte al estilo de vida de cada paciente y que, a su vez, permita ser replanteado en cada fase del proceso.

No hay diferencia de edad en la asistencia a la consulta médica especializada, lo cual indica que no hay edad específica para la asistencia a tratamientos de sobrepeso y obesidad, puesto que esta enfermedad, junto con sus manifestaciones clínicas, es posible padecerla en cualquier momento de la vida.

Lo mismo ocurre en cuanto al estado civil y a la ocupación, aunque se observó predominio entre estado civil casada y de ocupación finanzas y administración. Se evidencia que el sobrepeso y la obesidad se encuentran ampliamente distribuidas en toda la población.

En el estudio de Li et al. (22) se determinó la prevalencia del sobrepeso y obesidad en una población que se encontraba entre $18-79$ años de edad y se determinó el efecto negativo que presentaba el nivel educativo en cuanto a la correcta percepción del peso y la adopción de medidas para disminuirlo y, como analizaba este autor, exigía medidas en múltiples niveles para disminuir o controlar la epidemia de sobrepeso y obesidad entre los adultos de la ciudad de Beijing. De acuerdo con lo anterior, es importante tener en cuenta el nivel educativo y cultural de la población objeto de estudio. 
Según los datos del estudio, la muestra de pacientes analizados se encuentra en los estratos 3, 4 y 5 de una ciudad colombiana que se encuentra en crecimiento y que, en la actualidad, tiene aproximadamente un millón de habitantes, con una gran influencia comercial por ser ciudad fronteriza, lo que la hace tener características económicas bien particulares pero que, desde el punto de vista social, de salud $\mathrm{u}$ otras razones culturales, motiva a los pacientes a asistir a una consulta especializada de tipo privado, ya que han perdido la confianza en tratamientos, dietas e incluso cirugías, fenómeno al que se suma que el sistema de salud en Colombia no contempla alternativas de prevención y tratamiento que permitan hacer un seguimiento constante a los pacientes con sobrepeso u obesidad en el régimen contributivo y/o subsidiado.

Un estudio llevado a cabo en India por Gouda y Prusty (23) muestra un incremento notable de padecer sobrepeso y obesidad en los hogares no pobres, lo cual, como dicen los autores, lleva a la obesidad a convertirse en una epidemia urbana, a la cual se le debe dar la misma importancia que a las epidemias vistas en el pasado. Mientras tanto en Inglaterra, según Howel et al. (24), no se ve diferencia significativa de prevalencia y obesidad entre los diferentes estratos socioeconómicos.

También es importante, de manera significativa, la presencia de pacientes que previo a estos tratamientos, se han sometido a cirugías relacionadas con moldeamiento (p.e. liposucción y/o dermolipectomia). Esto se debe, en parte, a que estas pacientes ya no desean realizarse más cirugías y, en algunos casos, se encuentran con una autoestima baja debido a que han recuperado el peso que habían perdido en virtud del procedimiento quirúrgico.

Mercadenti y de Abreu (25) explican los efectos de la lipectomía (procedimiento en el cual se extirpa el tejido adiposo subcutáneo), como alternativa para mejorar el perfil metabólico a través de la rápida pérdida de tejido adiposo, pero, al no estar asociado a trastornos metabólicos como la resistencia a la insulina y diabetes mellitus tipo 2, se genera un crecimiento compensatorio de este tejido en respuesta a la lipectomía.

Sieffert et al. (26) determinaron que los pacientes obesos que se someten a procedimiento de cirugía plástica ambulatoria (liposucción, abdominoplastia, reducción de senos y blefaroplastia), incurren sustancialmente en mayores gastos de atención médica, en gran parte atribuible a los efectos adversos más frecuentes y el cuidado de la salud que debe llevarse a cabo en el hospital dentro de los 30 días posteriores a la cirugía.

Así mismo, Cuomo et al. (27), en su estudio sobre abdominoplastia, encuentran una mayor probabilidad de complicaciones después de la cirugía en pacientes obesos que tienen un nivel bajo de adiponectina, aunque en los demás pacientes el procedimiento quirúrgico es seguro y mejora la calidad de vida. De ahí la importancia de exámenes de laboratorio previos a cirugías plásticas, con el fin de determinar el riesgo en el postoperatorio.

Siendo el sobrepeso y la obesidad en la población mundial un peligro para la salud, es importante entrar a analizar el costo social perdido en materia de productividad y mortalidad prematura, que afecta la atención hospitalaria y la prescripción de medicamentos, según estudios realizados en Irlanda (28-29) y España (30).

Se hace necesario, en Colombia, establecer políticas más eficaces en materia de obesidad, que permitan prevenir la enfermedad con el único fin de evitar las consecuencias que, a corto y largo plazo, afectan la calidad de vida de los individuos que la llegan a padecer.

\section{CONCLUSIONES}

Se observó un predominio del género femenino en la asistencia a este tipo de consulta, que puede deberse no solamente a la preocupación por la belleza sino también a la capacidad de autocuidado que, de manera instintiva, se presenta en este género, lo cual incluye mantenerse en un buen estado de salud.

El estado civil casado y la ocupación hogar, junto con las finanzas y administración, se encontraron entre las características que más predominan en este tipo de pacientes. Sin embargo, se puede concluir que cualquier persona, independientemente de su estado civil y su actividad laboral, puede asistir a este tipo de tratamientos, presente o no manifestaciones clínicas de sobrepeso y obesidad, así como sus complicaciones.

(n)


ISSN 1794-9831

E-ISSN 2322-7028

Vol. 13 No. 1

Ene - Jun 2016

Cúcuta, Colombia

Tulia Isabel Copete-Villa, Pastor Ramirez-Leal

Dentro de las características clínicas llamó la atención la asistencia de pacientes que previamente se han realizado cirugías de moldeamiento tales como liposucción, dermolipectomía e inclusive las dos al mismo tiempo. Esto puede deberse, en gran parte, a que los pacientes han ganado el peso perdido como consecuencia de no presentar hábitos de alimentación y ejercicio adecuados para mantener el peso. Adicionalmente, las condiciones fisiológicas y de metabolismo pueden jugar un papel importante en el post-operatorio cuando no se tienen en cuenta entre los aspectos clínicos que requieren un seguimiento por parte del médico tratante en cada paciente.

Así mismo, para el médico tratante es de suma importancia conocer algunos hábitos que pueden orientar el tratamiento, tales como determinar si se presenta el hábito de ejercicio. En el primer control se evidencia que más del $50 \%$ de los pacientes no tiene el hábito del ejercicio. Teniendo en cuenta esta información se precisa a tomar la guía de tratamiento adecuada para cada paciente, la cual puede generar beneficios a corto, mediano o largo plazo, según sea la capacidad de incluir dentro de la rutina el hábito del ejercicio, junto con la dieta y demás procedimientos sugeridos en el tratamiento.

Entre los antecedentes clínicos de importancia se puede evidenciar la necesidad de asistencia a este tipo de tratamientos debido a múltiples enfermedades, entre las cuales predominan la dislipidemia y la hipertensión arterial como afectaciones que, de no ser tratadas a tiempo, pueden generar complicaciones graves para la salud de los pacientes.

Con la investigación se determinó la importancia de la intervención en los hábitos y estilo de vida de los pacientes que asisten a consulta para tratamiento integral de sobrepeso y obesidad, lo cual permite evidenciar avances significativos en la disminución del porcentaje de variación de peso y de variación del índice de masa corporal, especialmente en largos periodos de tiempo.

Los pacientes, por sí mismos, logran el mantenimiento de su peso en periodos cortos de tiempo, inclusive si se exponen a épocas del año donde, por razones de tradición y cultura, se acostumbra al consumo de alimentos con alto contenido calórico. Sin embargo, estos pacientes no deben dejar de asistir al control posterior programado, puesto que la obesidad es una enfermedad crónica que requiere la atención constante de un médico especializado que permita verificar los resultados del tratamiento y posibles alternativas que permitan alcanzar el peso ideal.

\section{CONFLICTO DE INTERESES}

Los autores declaran no tener ningún conflicto de intereses. 


\section{REFERENCIAS BIBLIOGRÁFICAS}

\section{Organización Mundial de la Salud (OMS). Obesidad y Sobrepeso. Centro de Prensa de la OMS, Nota descriptiva} No. 311. [Internet]. 2015 [consultado 16 de febrero de 2016] disponible en http://www.who.int/mediacentre/ factsheets/fs311/es/

2. Lastra-Lastra G, Lastra-González G. Obesidad: epidemia del nuevo milenio. Rev.fac.med. [Internet]. 2005 [consultado 18 de mayo de 2015];53(3):186-195. Disponible en: http://www.scielo.org.co/scielo.php?script=sci arttext\&pid=S0120-00112005000300005

3. Organización Panamericana de la Salud (OPS). Diabetes: OPS urge luchar contra la diabetes y la malnutrición en las Américas. [Internet]. 2008 [consultado 7 de junio de 2013] Disponible en: http://www.paho.org/hq/ index.php?option=com_content\&view=article\&id=405\%3A2008-diabetes-ops-urge-luchar-contra-obesidadmalnutricion-americas\&catid=740\%3Anews-press-releases\&Itemid=1926\&lang=es

4. Gómez-Dantés H. Páginas de Salud Pública. Salud pública de México. [Internet]. 2006 [consultado 19 de mayo de 2015]; 48(3):286-287. Disponible en: http://www.scielo.org.mx/pdf/spm/v48n3/29743.pdf

5. Pereira-Despaigne OL, Palay-Despaigne MS. Importancia de la reducción de peso en los pacientes con obesidad. MEDISAN [Internet]. 2015 [consultado 16 de febrero de 2016]; 19(8): 1043-1050. Disponible en: http://scielo. sld.cu/pdf/san/v19n8/san13198.pdf

6. DeMarco VG, Aroor AR, Sowers JR. The pathophysiology of hypertension in patients with obesity. Nat Rev Endocrinol [Internet]. 2014 [consultado 17 de febrero de 2016]; 10: 364-376. Disponible en: http://www.nature. com/nrendo/journal/v10/n6/abs/nrendo.2014.44.html

7. Herrera-Covarrubias D, Coria-Avila GA, Fernández-Pomares C, Aranda-Abreu GE, Manzo-Denes J, Hernández ME. La obesidad como factor de riesgo en el desarrollo de cáncer. Rev Peru Med Exp Salud Pública [Internet]. 2015 [consultado 16 de febrero de 2016]; 32 (4): 766-776. Disponible en: http://www.scielosp.org/scielo. php?script $=$ sci_arttext\&pid=S1726-46342015000400021\&lang=pt

8. Secretaría de Salud de Santander. Factores de riesgo para enfermedades crónicas en Santander, método STEPwise. [Internet]. 2011 [consultado 7 de junio de 2013]. Disponible en: http://www.who.int/chp/steps/2010_ STEPS_Survey_Colombia.pdf

9. Amzallag W. De perder peso, al control del peso. Rev Cubana Invest Bioméd [Internet]. 2000 [consultado 15 de febrero de 2015]; 19 (2): 98-115. Disponible en: http://scielo.sld.cu/scielo.php?script=sci_arttext\&pid=S086403002000000200002\&lng=es

10. Warburton DE, Nicol CW, Bredin SS. Health benefits of physical activity: the evidence. CMAJ [Internet]. 2006 [consultado 15 de febrero de 2016]; 174(6): 801-809. Disponible en: http://www.cmaj.ca/content/174/6/801. short

11. Malik VS, Willet WC, Hu FB, Global obesity: trends, risk factors and policy implications. Nat Rev Endocrinology [Internet]. 2013 [consultado 17 de febrero de 2016]; 9(1):13-27. Disponible en: http://www.nature. com/nrendo/journal/v9/n1/full/nrendo.2012.199.html

12. Ministerio de la Protección Social. Instituto Colombiano de Bienestar Familiar. Encuesta Nacional de la Situación Nutricional en Colombia [Internet]. Bogotá: Da Vinci Editores; 2011 [consultado 17 de febrero de 2016] Disponible en: http://www.icbf.gov.co/portal/page/portal/PortalICBF/Bienestar/ENSIN1/ENSIN2010/ LibroENSIN2010.pdf

13. Congreso de Colombia. Ley 1355 de 2009, octubre 14, por medio de la cual se define la obesidad y las enfermedades crónicas no transmisibles asociadas a ésta como una prioridad de salud pública y se adoptan medidas para su control, atención y prevención. [Internet]. Bogotá: Gobierno Nacional de Colombia; 2009 [consultado 17 de febrero de 2016] Disponible en: http://www.ins.gov.co:81/normatividad/Leyes/LEY\%20 $1355 \% 20 \mathrm{DE} \% 202009$.pdf

14. Martínez-Riquelme A, Sajoux I, Fondevila J. Resultados del estudio PROMESA I: eficacia y seguridad de la aplicación de una dieta de muy bajo aporte calórico y reeducación alimentaria posterior mediante el método Pronokal ${ }^{\circledR}$ en el tratamiento del exceso de peso. Nutr. Hosp [Internet]. 2014 [consultado 17 de febrero de 2016]; 29 (2): 282-291. Disponible en: http://scielo.isciii.es/scielo.php?script=sci_arttext\&pid=S0212$16112014000200006 \& \operatorname{lng}=\mathrm{es}$

15. Xiao L, Yank V, Wilson S, Lavori P, Ma J. Two- year weight-loss maintenance in primary care based diabetes prevention program lifestyle interventions. Nutr Diabetes [Internet]. 2013 [consultado 15 de febrero de 2015]; 3:e76. Disponible en: http://www.ncbi.nlm.nih.gov/pmc/articles/PMC3697405/

16. Yank V, Xiao L, Wilson SR, Safford RS, Rosas LG, Ma J. Short.term weight loss patterns, baseline predictors, and longer- term follow- up within a randomized controlled trial. Obesity [Internet]. 2014 [consultado 15 de 
febrero de 2015]; 22 (1): 45-51. Disponible en: http://onlinelibrary.wiley.com/doi/10.1002/oby.20510/pdf

17. Martínez-Hervaz S. Perímetro de cintura y factores de riesgo cardiovascular. Revista Española de Obesidad 2008; 6(2):97-104.

18. Moreno-González MI. Circunferencia de cintura: una medición importante y útil del riesgo cardiometabólico. Rev Chil Cardiol [Internet]. 2010 [consultado 17 de febrero de 2016]; 29: 85-87. Disponible en: http://www.scielo.cl/ $\mathrm{pdf} / \mathrm{rchcardiol} / \mathrm{v} 29 \mathrm{n} 1 / \mathrm{art} 08 . \mathrm{pdf}$

19. Organización Mundial de la Salud. Pautas para la Buena Práctica Clínica (BPC) en ensayos con productos farmacéuticos. Serie de Informes Técnicos de la OMS. No 850. [Internet]. 1995 [consultado 6 de marzo de 2016]. Disponible en: http://apps.who.int/medicinedocs/es/d/Jh2957s/\#Jh2957s

20. Johansson K, Neovius M, Hemmingsson E. Effectso of antiobesity drugs, diet, and exercise on weight loss maintenance after a very- low-calorie diet or low calorie diet: a systematic review and meta-analysis of randomized controlled trials. The American Journal of Clinical Nutrition [Internet]. 2014 [consultado 17 de febrero de 2016]; 99(1): 14-23. Disponible en: http://www.ncbi.nlm.nih.gov/pmc/articles/PMC3862452/

21. El ejercicio físico ligero disminuye el riesgo de obesidad y diabetes tipo 2. Rev Panam Salud Pública [Internet]. 2003 [consultado 19 de mayo de 2015]; 13(5):333-333. Disponible en: http://www.scielosp.org/scielo. php?script $=$ sci_arttext\&pid $=$ S1020-49892003000400010\&lng=en

22. Li C, Xiaoyan H, Zhi Q, Zhe L, Yumei Z, Peiyu W, et al. Prevalence of Overweight and Obesity and Weight Loss Practice among Beijing Adults, 2011. PloS One [Internet]. 2014 [consultado 17 de febrero de 2016]; 9(9): e98744. Disponible en: http://journals.plos.org/plosone/article?id=10.1371/journal.pone.0098744

23. Gouda J, Prusty RK. Overweight and obesity among women by economic stratum in urban India. J Health Popul Nutr [Internet]. 2014 [consultado 17 de febrero de 2016]; 32(1):79-88. Disponible en: http://www.ncbi.nlm.nih. gov/pmc/articles/PMC4089075

24. Howel D, Stamp E, Chadwick TJ, Adamson AJ, White M. Are social inequalities widening in generalised and abdominal obesity and overweight among English adults? PloS One [Internet]. 2013 [consultado 17 de febrero de 2016]; 8(11):e79027. Disponible en: http://www.plosone.org/article/fetchObject.action?uri=info:doi/10.1371/ journal.pone.0079027\&representation $=\mathrm{PDF}$

25. Mercadenti A, de Abreu-Silva EO. Different adipose tissue depots: Metabolic implications and effects of surgical removal. Endocrinol Nutr [Internet]. 2015 [consultado 17 de febrero de 2016]; 62(9): 458-64. Disponible en: http://www.ncbi.nlm.nih.gov/pubmed/26300495

26. Sieffert MR, Fox JP, Abbott LE, Johnson RM. Obesity is associated with increased health care charges in patients undergoing outpatient plastic surgery. Plas Reconstr Surg [Internet]. 2015 [consultado 17 de febrero de 2016]; 135(5):1396-404. Disponible en: http://paper.medlive.cn/literature/1273985

27. Cuomo R, Russo F, Sisti A, Nisi G, Grimaldi L, Brandi C, D’Aniello C. Abdominoplasty in Mildy Obese Patients (BMI 30-35 kg/m²): Metabolic Biochemical and Complication Analysis at one year. In Vivo [Internet]. 2015 [consultado 17 de febrero de 2016]; 29(6):757-61. Disponible en: http://www.ncbi.nlm.nih.gov/ pubmed/26546533

28. Dee A, Callinan A, Doherty E, O'Neill C, MacVeigh T, Sweeney MR, et al. Verweight and obesity on the island of Ireland: an estimation of costs. BMJ Open [Internet]. 2015 [consultado 17 de febrero de 2016];5:e006189. Disponible en: http://bmjopen.bmj.com/content/5/3/e006189.full.pdf + html

29. Dee A, Kearns k, O'Neill C, Shap L, Staines A, O'Dwyer V, et al. The direct and indirect costs of both overweight and obesity: a systematic review. BMC Res Notes [Internet]. 2015 [consultado 17 de febrero de 2016]; 7:242. Disponible en: http://bmcresnotes.biomedcentral.com/articles/10.1186/1756-0500-7-242

30. Mora T, Gil J, Sicras A. The influence of obesity and overweight on medical costs: a panel data perspective. Eur j Health Econ [Internet]. 2015 [consultado 17 de febrero de 2016]; 16(2): 161-73. Disponible en: https://www. researchgate.net/publication/259825979_The_influence_of_obesity_and_overweight_on_medical_costs_a_panel_ data_perspective 